\title{
50 años de planificación familiar en Chile, 1965-2015. Experiencias de las primeras generaciones de mujeres urbanas usuarias del programa
}

\author{
Patricia Castañeda M., $P h D^{1 a}$, Ana María Salamé C., $P h D^{2 b}$ \\ ${ }^{1}$ Escuela de Trabajo Social, Universidad de Valparaíso. ${ }^{2}$ Departamento de Trabajo Social, Universidad de La Frontera. \\ a Trabajadora Social.
}

\section{RESUMEN}

Antecedentes: En el año 1965, el Servicio Nacional de Salud chileno comenzó la ejecución directa de acciones sanitarias destinadas a implementar programas de planificación familiar, evento considerado hito fundacional que inició formalmente una política nacional de planificación familiar de carácter público y con respaldo gubernamental. Objetivo: Analizar la experiencia de las primeras generaciones de mujeres urbanas usuarias del programa de planificación familiar durante la década de 1960. Método: Estudio cualitativo con enfoque biográfico, realizado en 64 mujeres adultas mayores urbanas pertenecientes a las primeras generaciones usuarias del programa. Para la recolección de datos se utilizaron entrevistas con enfoque biográfico. Resultados: Se identificaron cuatro dimensiones: contexto familiar y social de la sexualidad femenina en infancia y adolescencia; proyecciones frente a la maternidad; experiencia personal de la usuaria en el programa y valoraciones de la usuaria en torno al programa. El programa impulsó el ejercicio del derecho de la mujer de decidir libremente y sin presiones el número de hijos que deseaba procrear. No obstante, por ser un derecho inédito hasta ese momento, su ejercicio fue instalándose progresivamente desde una construcción cultural de maternidad que no poseía dominio sobre sus eventos reproductivos, hacia una nueva construcción cultural que reconocía incipientemente mayores grados de decisión en la propia vida reproductiva. Conclusión: Se proponen tres perfiles tipo que ilustran las experiencias de las mujeres usuarias a partir de los componentes condición de fertilidad probada como requisito para el ingreso al programa de planificación familiar, número de hijos totales y espaciamiento entre nacimientos.

\section{PALABRAS CLAVE: Planificación familiar, derechos reproductivos}

\section{SUMMARY}

Background: In 1965, the Chilean National Health Service began the direct implementation of sanitary measures to implement family planning programs; considered foundational milestone event formally launched a national family planning policy of public and government-backed. Aims: To analyze the experience of the first generation of urban women users of family planning program during the 1960s. Method: Qualitative study with biographical approach, conducted in 64 elderly women in urban users belonging to the first generations of the program users. For data collection interviews were used to biographical approach. Results: We identified four dimensions: family and social context of female sexuality in childhood and adolescence; projections toward motherhood; personal experience of the user in the program and ratings of the user around the program. The program promoted the exercise of the right of woman to decide freely and without pressure the number of children they wanted to procreate. However, being an unpublished right so far, the exercise was settled progressively from a cultural construction of motherhood did not have control over their reproductive events, to a new cultural construction incipient recognized higher levels of decision itself 
reproductive life. Conclusion: It propose three kinds profiles that illustrate the experiences of women users from components proven fertility status as a requirement for admission to the program of family planning, total number of children and birth spacing.

\section{KEY WORDS: Family planning, reproductive rights}

\section{INTRODUCCIÓN}

En el año 1965, el Servicio Nacional de Salud chileno comenzó acciones sanitarias destinadas a implementar programas de planificación familiar, asumiendo la responsabilidad de aplicar con eficacia técnica los métodos anticonceptivos en la población femenina del país. Esta decisión institucional se considera hito fundacional que inició formalmente una política nacional de planificación familiar de carácter público con respaldo gubernamental (1). Sobre dicha base se formuló en 1967 la Política de Población y de Salud Pública que planteaba tres desafíos: i) reducir la tasa de mortalidad materna por aborto provocado que alcanzaba a 279 defunciones por cada 100 mil nacidos vivos, representando el $40 \%$ de las muertes maternas; ii) reducir la tasa de mortalidad infantil que alcanzaba a 120 por mil nacidos vivos, vinculada con el bajo nivel de vida de la población con alta fecundidad y iii) promover el bienestar familiar, favoreciendo el ejercicio del derecho a una paternidad responsable (2).

A cinco décadas de dicho hito, las estadísticas vitales nacionales muestran el impacto de su implementación. La tasa de mortalidad materna ha descendido desde 118 muertes maternas por 100 mil nacidos vivos en 1964 a 20 muertes maternas en el año 2000. La tasa global de fecundidad ha descendido desde 5,4 hijos promedio por mujer en 1960 a 1,9 en el año 2010. La fecundidad del país descendió un $65 \%$ en cinco décadas y no se aprecian factores internos que alteren la tendencia en el futuro. En forma complementaria, en 1960 el 3,4\% de las mujeres tenía estudios universitarios, cifra que sube en la actualidad al $25 \%$. Asimismo, en 1960 las mujeres representaban el $21 \%$ de la fuerza laboral chilena, incrementándose a $46,6 \%$ en el año 2010 (3).

Teniendo como marco la conmemoración de los cincuenta años de la planificación familiar en Chile, el objetivo de este estudio es analizar la experiencia de las primeras generaciones de mujeres urbanas usuarias del programa de planificación familiar durante la década de 1960.

\section{SUJETOS Y MÉTODOS}

Estudio cualitativo de enfoque biográfico, realizado en 64 mujeres adultas mayores urbanas, usuarias de servicios públicos de salud de las regiones de Valparaíso y Araucanía. La muestra cua- litativa posee representatividad estructural intencionada definiendo como sujeto a mujeres adultas mayores urbanas que durante su edad fértil, participaron como primeras generaciones usuarias del programa de planificación familiar en la década de 1960. Los criterios de inclusión correspondieron a: mujer usuaria del programa de planificación familiar en la década definida para el estudio, en condiciones de salud mental que permitiesen la entrega de un relato coherente de su experiencia y que participara del estudio libre y voluntariamente. La estrategia de recolección de información correspondió a entrevista cualitativa con enfoque biográfico, definida como una narrativa centrada en eventos vitales con fines de análisis científico (4). Las entrevistas fueron realizadas en domicilio entre los meses de mayo a octubre del año 2013. El día de la entrevista se solicitó leer y firmar un consentimiento informado, que explicaba las características del estudio y sus derechos como sujetos de investigación. El guión temático estuvo organizado en torno a preguntas abiertas asociadas a las siguientes dimensiones definidas para el estudio: i. Contexto familiar y social en torno a la sexualidad femenina en las etapas de infancia y adolescencia, ii. Proyecciones frente a la maternidad, iii. Experiencia personal de usuaria del programa de planificación familiar durante la edad fértil, y iv. Valoraciones de la usuaria respecto a su experiencia en el programa de planificación familiar.

Las entrevistas tuvieron un promedio de $90 \mathrm{mi}-$ nutos de duración y fueron grabadas en audio y transcritas in extenso para fines de análisis cualitativo. Los relatos fueron segmentados en unidades significativas y organizados en sistemas de categorías. Los criterios de rigor que garantizan validez y confiabilidad del estudio correspondieron a recogida de abundante información, desarrollo de descripciones minuciosas y ejercicio de reflexión teórica permanente. Se logró la saturación de los datos en la entrevista 16 , sin embargo, se continúo con la programación previa para cumplir con los criterios de rigor definidos. Esta investigación se adscribe a los protocolos de vigilancia ética establecidos en la Declaración de Helsinki de 1975 revisada en 1983.

\section{RESULTADOS}

Se entrevistó a un total de 64 mujeres adultas mayores urbanas, con edad promedio de 69,9 años (rango: 60 - 81 años) y promedio de hijos vivos 3,4 
por mujer (rango: 2 - 7 hijos vivos). El promedio de años de relación matrimonial o de pareja alcanzó a 48,8 años (rango: 37 - 61 años). El método anticonceptivo de mayor referencia correspondió a dispositivos intrauterinos. Los relatos recogidos permiten identificar las siguientes dimensiones y subdimensiones asociadas (Tabla I).

La dimensión contexto familiar y social en torno a la sexualidad femenina en las etapas de infancia y adolescencia refiere una profunda desinformación respecto de los eventos reproductivos. "Yo aprendí sola. A mi nadie me explicó lo que es el matrimonio. Bueno, antes era así la cuestión. Se tenían las guaguas y listo. Nosotros éramos ignorantes y no teníamos quien nos explicara".

Asimismo, se evoca una rígida diferenciación de roles y actividades por género en las responsabilidades de cada integrante de la familia. "La mamá se dedicaba a las tareas de la casa, el cuidado de los niños y el papá salía a trabajar. Las niñas pasábamos más tiempo con mi mamá y ayudábamos en las tareas del hogar. Los hijos le ayudaban al papá cuando estaba en casa y no salía a trabajar, o en la agricultura o en otra cosa"

La diferenciación de roles también se presentaba en el plano sexual, donde las mujeres refieren mayores restricciones respecto de los hombres. "El varón podía tener por ahí sus aventuras, pero la niña no podía. Las mamás le metían miedo a las hijas, que el marido recién casado le iba a notar que ella habría tenido actividad sexual y eso hacía que la mujer se conservara siempre virgen".

En relación a la dimensión proyecciones frente a la maternidad, el discurso evidencia resignación respecto al embarazo y el número de hijos totales. "Nunca hablamos con mi esposo de ese tema. Nunca propusimos el número de hijos que decidíamos tener en la familia. Los niños Ilegaban porque tenían que llegar no más"

No obstante lo anterior, emerge un discurso subsumido de estrategias de anticoncepción e interrupción de embarazos que manejaban las mujeres antes del inicio del programa de planificación familiar. "El aborto se practicaba mucho. Todas las mujeres que no querían tener la cantidad de hijos que tenían nuestras abuelas, que eran catorce, quince hijos, se hacian abortos. Iban donde una persona que supiera, alguna podría ser matrona y otras señoras que se dedicaban a realizar esa operación. Era ir donde una persona que supiera hacer eso $y$ el precio estaba de acuerdo a los ingresos de ellos". "Entre amigas se contaba que existían los pesarios, que eran como de vaselina dura, se ponían antes de la relación y eso impedía que hubiera fecundación. Eran del tamaño de una cucharita chica y se ponía en la vagina antes de la relación".

\section{Tabla I \\ DIMENSIONESY SUBDIMENSIONES EXTRAÍDAS DE LAS ENTREVISTAS DE LAS PRIMERAS GENERACIONES USUARIAS DE LA POLÍTICA DE PLANIFICACIÓN FAMILIAR}

DIMENSIÓN
Contexto familiar y social en torno a la sexualidad fe-
menina en las etapas de infancia y adolescencia

menina en las etapas de infancia y adolescencia

Proyecciones frente a la maternidad

Experiencia personal de la usuaria como del programa de planificación familiar durante la edad fértil

Valoraciones de la usuaria respecto de su experiencia en el programa de planificación familiar

\section{SUBDIMENSIONES}

- Desinformación respecto de la sexualidad.

- Diferenciación de roles masculino y femenino tradicionales.

- Diferenciación en el ejercicio de la sexualidad entre hombres y mujeres.

- Discurso socialmente esperado: Aceptación de los embarazos.

- Discurso subsumido. Estrategias alternativas de planificación familiar e interrupción del embarazo desde saberes femeninos intergeneracionales.

- Embarazo como evento de enlace.

- Valoraciones positivas/negativas respecto del equipo de salud.

- Valoraciones negativas respecto de la pareja. 
"Ella después de la relación se levantaba al tiro y se hacía un lavado vaginal, ella contaba que tuvo a sus hijos cuando ella quería"

Respecto de la dimensión experiencia personal de usuaria del programa de planificación familiar durante la edad fértil, se confirma que el factor central de enlace con el programa era el embarazo. "Yo conocí el programa cuando ya no había remedio y estaba embarazada. Yo me enteré por el consultorio, me dijeron que ahí habían empezado las pastillas, y después vinieron otros métodos como la $T$ de cobre"

Los discursos de las mujeres en la dimensión valoraciones de las usuarias respecto del programa, permite identificar valoraciones positivas y negativas respecto del equipo de salud y valoraciones negativas respecto de la participación de la pareja en el proceso. "El programa fue lo mejor que tuvimos, estábamos en el consultorio esperando que atendieran a la guagua y viene una señorita $y$ empieza a hablarnos de planificación familiar. Nosotros no sabíamos ni lo que era eso. Ella explicó que no nos iba a pasar nada, que nos iban a poner algo intrauterino. Todas estábamos encantadas de la vida, porque el temor de tener otro hijo cuando se sabía lo que venía, la miseria, otro hijo era la miseria, entonces no se podía tener muchos hijos, nosotros veníamos de familia de miseria. Todo era sano, atendidos por la medicina del país, era algo seguro. No desconfiamos de que fuera ilegal, de todas formas ya lo habian explicado ellas, como era, como íbamos a quedar bien, como no íbamos a padecer nada, que los síntomas que íbamos a tener. Pero que a veces fallaban también, que también existían riesgos". "Uno llegaba y le decían, a usted le van a poner el espiral, como obligación, no daban otras alternativas. Uno decía si quería colocárselo o no. Uno no decidía sobre que método usar, pero si podía decidir si usarlo o no". "En el consultorio tampoco eran de lo más amables, sobre todo las matronas mayores, aunque uno estuviera casada, igual un tema complicado". "Los hombres tenían una crianza muy machista y se negaban a que sus esposas tomaran anticonceptivos porque pensaban que podían ser engañados. Había mucho temor de dar cierta libertad a las mujeres a través de los anticonceptivos".

\section{DISCUSIÓN}

La información recopilada rescata las experiencias y valoraciones realizadas por las primeras generaciones de mujeres usuarias de la planificación familiar como política pública en núcleos urbanos regionales. En ese marco, las usuarias corresponden a mujeres formadas en su infancia desde roles masculinos y femeninos tradicionales, que definían su proyecto de vida como sujetos sexualmente pasivos, siendo la maternidad su realización vital central, conforme a la realidad cultural de la época.

Acorde a los planteamientos de Gomenzoro y Lutz (5) la experiencia confirma que el programa de planificación familiar permitió a las mujeres acceder en medio de su vida reproductiva y con fertilidad probada, al ejercicio del derecho de decidir libremente el número de hijos que deseaban procrear en su futuro inmediato. No obstante, por ser un derecho inédito, su ejercicio fue instalándose progresivamente desde una construcción cultural de maternidad que no poseía dominio sobre los eventos reproductivos, hacia una nueva concepción que reconocía incipientemente mayores grados de decisión en la propia vida reproductiva.

Este cambio no se presentó exento de dificultades derivadas por una parte, de las concepciones de sexualidad existentes en la mirada cultural masculina vigente en la época, que desconfiaba de los potenciales nuevos ámbitos de decisiones sexuales autónomas, que las mujeres obtenían como usuarias del programa y, por otra parte, desde las estrategias de atención desarrolladas por los equipos de salud. En este último aspecto, se produce una tensión con el concepto de planificación familiar revisado, que reconoce la necesidad que las personas reciban la información necesaria, objetiva, cierta y oportuna, así como educación sexual complementaria y facilidades para disponer, independientemente de su nivel económico y cultural, de la asistencia anticonceptiva adecuada.

La información recopilada sugiere que en ocasiones, el equipo de salud tomó unilateralmente las decisiones respecto a los métodos anticonceptivos, otorgando mayor preeminencia a los criterios de urgencia sanitaria frente a la problemática del aborto, por sobre la propia decisión informada de cada usuaria. No obstante, debe tenerse presente que las mayores oportunidades de información se veían afectadas por los bajos niveles de escolaridad de la población nacional, que en aquella época reportaban una tasa de analfabetismo que superaba el $25 \%$ (6).

El análisis realizado devela una tensión entre un discurso femenino socialmente esperado, de aceptación plena de la maternidad, que se entrelaza con un discurso subsumido que reconoce la existencia de saberes y prácticas femeninas transmitidas intergeneracionalmente en torno a estrategias de prevención o interrupción del embarazo. En ese marco, el aborto es referido como una actividad utilizada regularmente como recurso de interrupción del embarazo, situación coincidente con las preocupaciones sanitarias de la época, que lo situaban como la primera causa de muerte en la población 
femenina en edad fértil, afectando principalmente a mujeres casadas entre los 20 y 34 años, que tenían hasta 3 hijos vivos y que pertenecían a grupos socioeconómicos de menores ingresos (7). Estos antecedentes confirman que las mayores destinatarias de la planificación familiar resultaron ser las mujeres en pleno proceso reproductivo, que vieron modificarse estructuralmente su calidad de vida, al ser destinatarias de una política pública constituida por una oferta de servicios sanitarios oportunos, que desincentivaron las antiguas y riesgosas prácticas abortivas vigentes en la época. Actualmente, el aborto posee un peso marginal en la tasa global de mortalidad materna, demostrando el éxito de la política de planificación familiar en la materia $(8,9)$.

A partir del análisis realizado, y considerando la condición de fertilidad probada como requisito para el ingreso al programa de planificación familiar, el número de hijos totales y el espaciamiento entre nacimientos, pueden proponerse tres perfiles tipo que ilustran las experiencias de las primeras generaciones de mujeres usuarias del programa:

Perfil 1. Usuarias con fertilidad probada con número de hijos totales conforme al promedio de la tasa global de fecundidad de la época, ajustan el tiempo de espaciamiento entre nacimientos una vez incorporadas en el programa (Figura 1).
Perfil 2. Usuarias con fertilidad probada ajustan el número de hijos totales a un nivel inferior al promedio de la tasa global de fecundidad de la época y el tiempo de espaciamiento entre nacimientos, una vez incorporadas en el programa (Figura 2).

Perfil 3. Usuarias con fertilidad probada ajustan el número de hijos totales a un nivel inferior al promedio de la tasa global de fecundidad de la época al evitar nuevos embarazos una vez incorporadas en el programa (Figura 3).

\section{CONCLUSIÓN}

A contar de 1965, se observa una baja sostenida en la tasa global de fecundidad del país, junto con el aumento progresivo de la escolaridad femenina y de la participación de la mujer en el mundo laboral, confirmando que cinco décadas de políticas de planificación familiar han modificado estructuralmente las condiciones sanitarias y culturales de la población femenina chilena en torno a la maternidad, ofreciéndoles nuevas oportunidades de desarrollo personal y una mejor calidad de vida a partir del pleno ejercicio del derecho de decidir libremente y sin presiones el número de hijos e hijas que desean procrear.

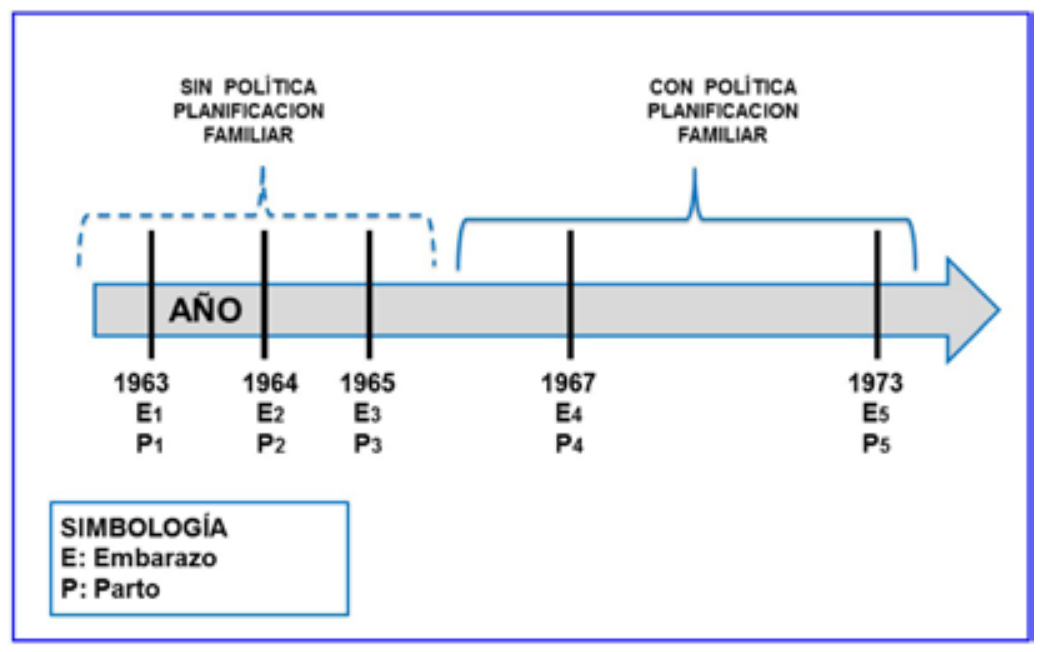

Figura 1. Perfil 1: Usuarias con fertilidad probada espacian nacimientos como resultado de incorporación al programa. Número de hijos totales en el promedio de la tasa de fecundidad de la época. 


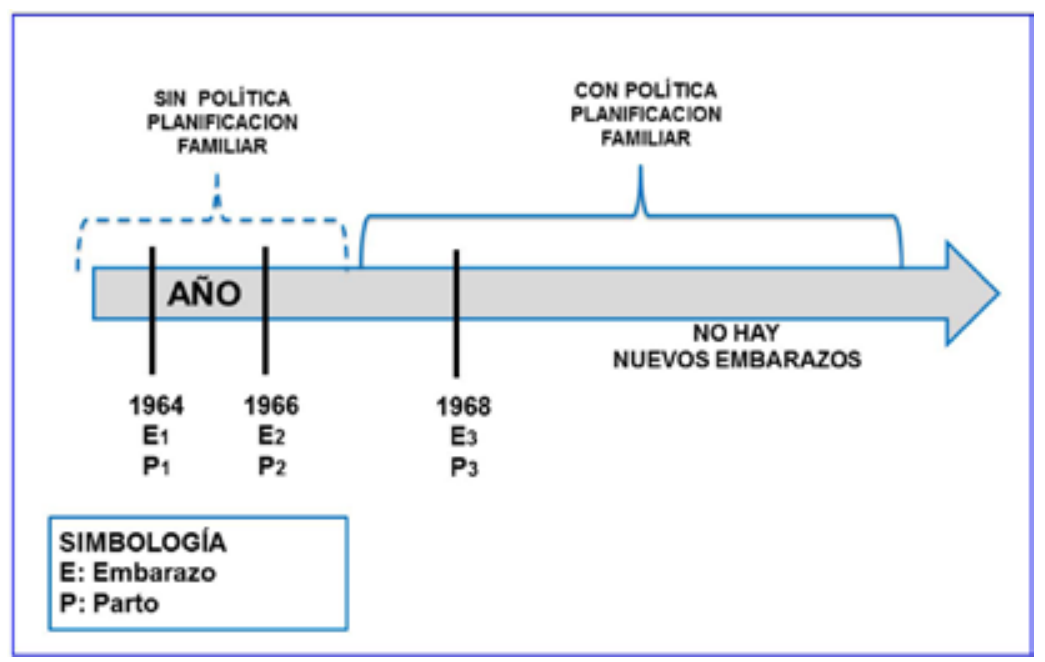

Figura 2. Perfil 2: Usuarias con fertilidad probada espacian nacimientos como resultado de incorporación al programa. Número de hijos totales inferiores a la tasa de fecundidad de la época.

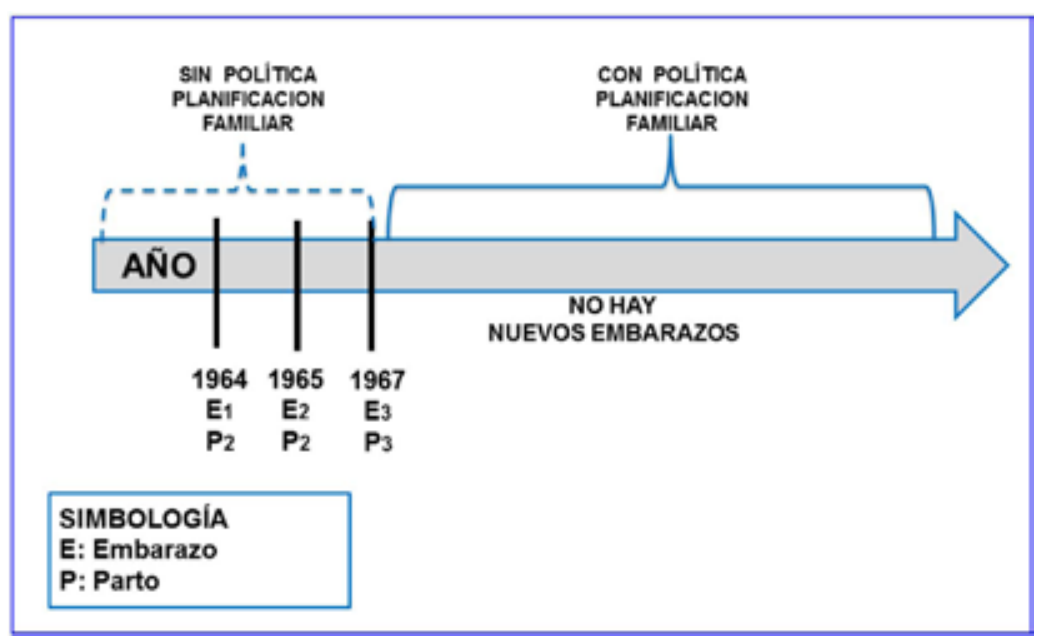

Figura 3. Perfil 3: Usuarias con fertilidad probada evitan un nuevo embarazo. Número de hijos totales inferiores al promedio de la tasa de fecundidad de la época. 


\section{REFERENCIAS}

1. Avendaño O. Desarrollo histórico de la planificación de la familia en Chile y en el mundo. Ediciones APROFA. Santiago de Chile 1975; pp 33-71.

2. Ministerio de Salud. Normas Nacionales sobre Regulación de la Fertilidad. MINSAL, Santiago de Chile 2008; pp 11-18.

3. Instituto Nacional de Estadísticas. Anuario de Estadísticas Vitales. Informe Anual 2012. Santiago de Chile 2012; pp 15-38.

4. Valles M. Entrevistas cualitativas. Centro de Investigaciones Sociológicas. Ediciones CIS. Serie Cuadernos Metodológicos. Madrid 2002; pp 28-34.
5. Gomensoro A, Lutz E. Anticoncepción. Aportes para una polémica constructiva. Biblioteca ISIS Internacional. Documento de Trabajo. Mimeo Santiago de Chile 1982; pp 35-8.

6. Ministerio de Salud, Normas Nacionales sobre Regulación de la Fertilidad. MINSAL, Santiago de Chile 2008. pp.10-21.

7. Szot J. Reseña de la salud pública materno-infantil chilena en los últimos 40 años: 1960-2000. Rev Chil Obstet Ginecol 2002;67(2):129-35.

8. Szot J, Moreno C. Mortalidad por aborto en Chile: análisis epidemiológico 1985-2000. Rev Chil Obstet Ginecol 2003:68(4):309-14.

9. Donoso E. Mortalidad materna en Chile: tras el cumplimiento de una meta. Rev Chil Obstet Ginecol 2002;67(1):44-6. 